\title{
KBV-Vertreter setzen Aufklärungsgremium ein
}

_ Die Vertreterversammlung (VV) der KBV hat in ihrer Sondersitzung am 30. Oktober 2015 die Gründung eines Vertrauensausschusses beschlossen. Dieser soll Verfahrens- und Verhaltensempfehlungen an die Mitglieder der VV erarbeiten, wie mit den aufgetretenen kontroversen Themen umgegangen werden könnte. Dem Ausschuss soll neben sechs VV-Mitgliedern auch ein externer Beauftragter angehören, der als Berater juristische Expertise haben muss.

\section{MMW-KOMMENTAR}

Konkret geht es um Mietzuschüsse an den ehemaligen Vorsitzenden des KBV-Vorstandes, Dr. Andreas Köhler, und Pensionszusagen an ihn und andere Mitarbeiter der KBV, die nicht hätten gezahlt bzw. gemacht werden dürfen. Offene Fragen gibt es auch noch bei den finanziellen Transaktionen um den Bau der KBV-Immobilien in Berlin.

Da die KBV in diesen Fragen bisher Tendenzen zeigte, auf Zeit zu spielen und die Probleme auszusitzen, hatte die Aufsichtsbehörde bereits im Vorfeld der Sitzung reagiert. Mit Schreiben vom 12. September hatte das Bundesgesundheitsministerium der KBV eine letzte Chance gegeben, bis zur nächsten regulären Sitzung der VV am 4. Dezember ihre Beratungen abzuschließen und über das weitere Vorgehen zu entscheiden. Sollte es nicht gelingen, bei der Sonder-VV am 30. Oktober einen arbeitsfähigen Vertrauensausschuss zu installieren, müsse man eine andere Lösung finden.

Das Ministerium erwartet nun eine abschlieBende Beschlussfassung der VV und eine Stellungnahme des Vorstandes zum weiteren Vorgehen bis zum 7. Dezember. Ansonsten will es selbst im Rahmen seiner Rechtsaufsicht die Zügel in die Hand nehmen. Der gerade gebildete Ausschuss hat also nicht viel Zeit, die offenen Fragen zu beantworten.

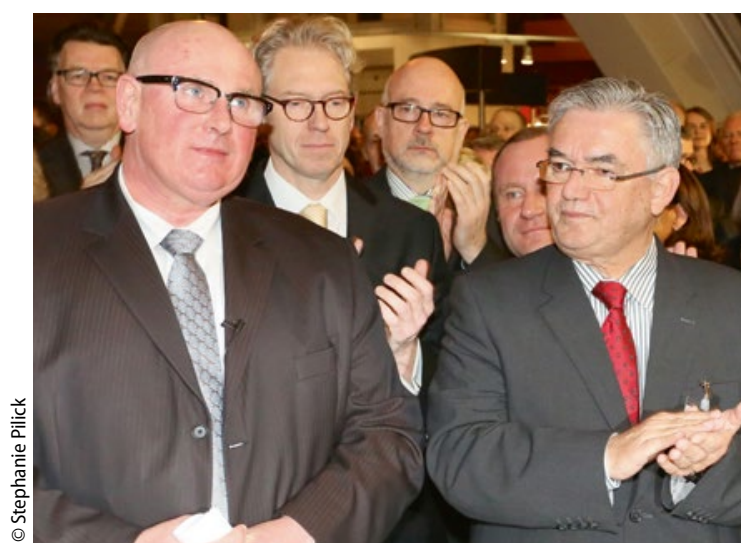

Kritische Connection: Ex-KV-Chef Köhler (I.) und der VV-Vorsitzende Weidhaas (r.).

\section{GOÄ: Sonderleistung neben Beratung abrechnen}

Die immer noch gültige GOÄ nennt zum Themenkomplex „Beratung und Sonderleistungen“ zwei wesentliche Einschränkungen, mit denen man sich bei der Abrechnung im privatärztlichen Bereich fast täglich auseinandersetzen muss. Gemäß Punkt 2 der Präambel zum Kapitel B, den Grund- und allgemeinen Leistungen, sind „die Leistungen nach den Nummern 1 und/oder 5 neben Leistungen nach den Abschnitten $\mathrm{C}$ bis $\mathrm{O}$ im Behandlungsfall nur einmal berechnungsfähig“. Des Weiteren kann die Nr. 3 GOÄ, das Gespräch von mindestens zehn Minuten Dauer, nur neben den Untersuchungsleistungen nach den Nrn. $5,6,7,8,800$ oder 801 angesetzt werden.

\section{MMW-KOMMENTAR}

Diese Einschränkungen gelten auch für das Kapitel M, also für Laboruntersuchungen.
Dies ist aber nur der Fall, wenn die Analysen während des aktuellen Arzt-Patienten-Kontaktes in der Praxis erfolgen, also im sogenannten Akutlabor. Denn nur bei gleichzeitiger Erbringung von Beratung und Laborleistung greift die unter Punkt 2 festgehaltene Vorgabe der Präambel zum Kapitel B, also in der Regel bei Schnelltests (Urin, Quick-Wert, INR, D-Dimere, Blutzucker etc.).

Neben den Nrn. 1 und 5 können die Laborleistungen zumindest beim ersten Kontakt im Behandlungsfall problemlos abgerechnet werden. Berücksichtigen muss man dabei, dass in der GOÄ der Behandlungsfall als der Zeitraum von einem Monat ab Behandlungsbeginn definiert ist. Bei weiteren Kontakten muss man dann rechnen. Ist die Beratung mit 80 Punkten besser bewertet als die durchgeführten Laboruntersuchungen? Typische Leistungen unterhalb von 80 Punkten sind die Blutsenkung (Nr. 3501), der Urin-
Teststreifen (Nr. 3511) oder die Blutzuckerbestimmung (Nr. 3514). Mit mehr als 80 Punkten bewertet ist hingegen der komplette Urinstatus (Nrn. 3511 + 3531) oder die INR-Bestimmung (Nr. 3530).

Ergibt sich eine Kombination aus der Nr. 3 und Sonderleistungen, so kann man die Bestimmungen des $\S 5$ der GOÄ zugrunde legen. Danach ist es möglich, bei entsprechendem Aufwand einen höheren Multiplikator zum Ansatz zu bringen. Da die Leistungen nach den Nrn. 1 und 5 beim ersten Kontakt im Behandlungsfall die Kombination mit den genannten Sonderleistungen ermöglicht, wäre eine Abrechnungsfolge $1+5$ (Multiplikator Z. B. 3 wegen besonderem zeitlichen Beratungs- und Untersuchungsaufwand) und z. B. die gleichzeitige Berechnung einer Ergometrie (Nr. 652) und Lungenfunktion (Nrn. 605, 605a) möglich. 